Original Paper http://ajol.info/index.php/ijbcs http://indexmedicus.afro.who.int

\title{
Utilisation du moringa, de la spiruline, de la patate douce à chair orange et d'un complexe minéral et vitaminique dans la fabrication de biscuits de sorgho enrichis destinés aux jeunes enfants
}

\author{
Laurencia Toulsoumdé SONGRE-OUATTARA ${ }^{1 *}$, Kourfom GORGA ${ }^{1}$, \\ Fabrice BATIONO $^{1}$, Aly SAVADOGO ${ }^{2}$ et Bréhima DIAWARA ${ }^{1}$
}

${ }^{1}$ Institut de Recherches en Sciences Appliquées et Technologies (IRSAT), Département Technologie Alimentaire (DTA), 03 BP 7047 Ouagadougou 03 Burkina Faso.

${ }^{2}$ Université Ouaga I Pr Joseph KI-ZERBO, Unité de formation et Recherche en Sciences de la Vie et de la Terre (UFR/SVT), Laboratoire LABIA 03 BP 7021 Ouagadougou 03, Burkina Faso.

*Auteur correspondant ; E-mail: laurenciaouattara@yahoo.fr, Tél: 0022670278633.

\section{REMERCIEMENTS}

Ces travaux ont été financés par le septième programme cadre de l'Union Européenne [Fp7/20072013 J à travers le projet INSTAPA (Novel staple food-based strategies to improve micronutrient status for better health and development in sub-saharan Africa) sous l'agrément $N^{\circ} 211484$.

\section{RESUME}

Les biscuits peuvent contribuer à améliorer le statut nutritionnel des jeunes enfants. L'effet de l'incorporation du moringa, de la spiruline, de la patate douce à chair orange (PDCO) et d'un complexe minéral et vitaminique (CMV) sur la qualité nutritionnelle des biscuits de sorgho est étudié. Pour ces ingrédients nutritionnels, trois niveaux d'incorporation sont étudiés à travers douze formulations de biscuit et leurs caractéristiques nutritionnelles et sensorielles analysées par des méthodes standard et comparées au biscuit témoin non enrichi. Les résultats obtenus montrent que les teneurs varient entre $1,8 \pm 0,6 \%$ et $7,9 \pm 1,1 \%$ pour l'humidité, entre $5,6 \pm 1,4 \%$ et $11,7 \pm 0,7 \%$ pour les protéines, entre $19,5 \pm 4,6 \%$ et $23,5 \pm 1,6 \%$ pour les lipides, entre $2 \pm 0,0 \%$ et $4,1 \pm 0,1 \%$ pour les cendres et entre $53,2 \pm 2,2 \%$ et $68,5 \pm 0,5 \%$ pour les glucides. L'augmentation des teneurs en protéines est significative avec l'addition du moringa et de la spiruline. La valeur énergétique est comparable pour l'ensemble des formulations avec cependant des profils nutritionnels différents. Les teneurs en fer, contrairement au zinc sont significativement plus élevées $(p<0,05)$ dans les biscuits de sorgho enrichis. En conclusion, les formulations de biscuits de sorgho les plus acceptables au plan nutritionnel et sensoriel sont celles enrichies à $4 \%$ de spiruline, $10 \%$ de moringa et $18 \%$ de PDCO.

(c) 2016 International Formulae Group. All rights reserved.

Mots clés : Biscuit de sorgho, moringa, spiruline, patate douce à chair orange, caractéristiques nutritionnelles.

\section{Use of the moringa, spirulina, orange-fleshed sweet potato and a multivitamin} complex in the manufacture of enriched sorghum biscuits for young children

\section{ABSTRACT}

Cookies can help to improve the nutritional status of young children. The effect of moringa, spirulina, orange fleshed sweet potato (PDCO) and multivitamin complex (CMV) incorporation on the quality of sorghum cookies was studied. For these nutritional ingredients, three rates of incorporation were studied through 12 cookies formulations and the nutritional and sensory characteristics analyzed using standard 
methods and compared with control cookies without incorporation. Results showed that the contents varied between $1.8 \pm 0.6 \%$ and $7.9 \pm 1.1 \%$ for humidity, $5.6 \pm 1.4 \%$ and $11.7 \pm 0.7 \%$ for protein, $19.5 \pm 4.6 \%$ and $23.5 \pm 1.6 \%$ for fat, $2.0 \pm 0.0 \%$ and $4.1 \pm 0.1 \%$ for the ashes and between $53.2 \pm 2.2 \%$ and $68.5 \pm 0.5 \%$ for carbohydrate The increase in protein levels was significant with moringa leaves powder and spirulina addition. The energy densities were similar for all formulations although the nutrient profiles were different. Fe contents, in the opposite of zinc were significantly higher $(\mathrm{p}<0.05)$ in the enriched sorghum cookies. In short, the more acceptable sorghum cookies formulations in regard to nutritional and sensorial aspects were those enriched with $4 \%$ of spirulina, $10 \%$ of moringa leaves powder and $18 \%$ of PCDO.

(C) 2016 International Formulae Group. All rights reserved.

Keywords: Cookies of sorghum, moringa, spirulina, orange fleshed sweet potato, nutritional characteristic.

\section{INTRODUCTION}

La malnutrition multi-carentielle, notamment celle due aux carences en micronutriments, y inclus le fer et le zinc, sont des problèmes de santé publique qui affectent les populations de tous les âges dans les pays en développement (PED) et plus particulièrement les jeunes enfants avec des conséquences physiologiques et économiques non négligeables (UNICEF, 2009). Au Burkina Faso, l'émaciation, le retard de croissance, l'insuffisance pondérale et l'anémie nutritionnelle touchent respectivement $16 \%, 35 \%, 26 \%$ et $88 \%$ des enfants de moins de 5 ans (INSD, 2011). Face à la malnutrition, plusieurs stratégies sont promues à l'échelle internationale par de nombreuses instances pour prévenir et lutter contre ce fléau. A cet effet, la complémentation qui consiste à l'identification d'un ou plusieurs aliments potentiellement intéressants sur le plan nutritionnel pour compléter un autre aliment en vue d'améliorer la qualité nutritionnelle de l'ensemble (Mensah et Tomkins, 2003) est suggérée comme une approche intéressante, et accessible à moindre coût pour lutter contre la malnutrition dans les PED (Anwar et al., 2007 ; Ndong et al., 2007). De nombreuses ressources végétales possèdent des potentiels nutritionnels qui leur permettent d'être de bon candidat, comme complément alimentaire ou nutritionnel pour lutter contre la malnutrition. Ainsi, le moringa (Moringa oleifera), la spiruline (Spirulina platensis) et la patate douce à chair orange (Ipomoea batatas) peuvent être utilisés pour compléter de nombreux aliments (Ndong et al., 2007; Thurber et Fahey, 2009).

Les feuilles de moringa sont une denrée alimentaire importante qui a une énorme attention dans la nutrition dans les pays tropicaux (Mawouma et Mbofung, 2014).Les feuilles de moringa sont rapportées être une riche source de protéines, B-carotène, vitamine $\mathrm{C}$, calcium et potassium et de nombreux antioxydants essentiels pour la nutrition et la santé de l'homme (Dillard et German, 2000; Siddhuraju et Becker, 2003; Answar et al., 2007) et souvent utilisées pour la prévention et la lutte contre la malnutrition en Afrique et en Asie (Tete-Benissan et al., 2012). Cent grammes de feuilles séchées de moringa contiennent $25 \mathrm{~g}$ de protéines, $8 \mathrm{~g}$ de lipides, $12 \mathrm{~g}$ de minéraux, $40 \mathrm{~g}$ de glucides, 15 $\mathrm{g}$ de fibres, $27 \mathrm{mg}$ de fer, 2,6 $\mathrm{mg}$ de zinc, 14300 UI de vitamine A, $850 \mathrm{mg}$ de vitamine C (Broin, 2005).

La spiruline qui est une micro-algue est considérée par l'organisation mondiale de la santé (OMS) comme un aliment extraordinaire pour ses grandes valeurs nutritives. Elle est produite et commercialisée à travers le monde et utilisée comme complément alimentaire chez les humains et les animaux (Belay, 2002 ; Muhling et al., 2005). Elle est une riche source de presque tous les éléments nutritionnels (Diraman et al., 2009; Holman et Malau-Aduli, 2013). Cent grammes de spiruline contiennent 4 à $9 \mathrm{~g}$ d'humidité, 4 à $16 \mathrm{~g}$ de lipides, 60 à $70 \mathrm{~g}$ de protéines, 3 à 11 $\mathrm{g}$ de matières minérales, 14 à $19 \mathrm{~g}$ de glucides totaux, $140 \mathrm{mg}$ de $\beta$-carotène, $1200 \mathrm{mg} / \mathrm{kg}$ de 
calcium (Sanchez et al., 2003; Babadzhanov et al., 2004; Habib et al., 2008).

La patate douce à chair orange (PDCO) est un tubercule qui fait l'objet de nombreux travaux sur des groupes d'individus depuis quelques années pour sa grande richesse en vitamine A (Haskell et al., 2004; van Jaarsveld et al., 2005). Selon la table de composition nutritionnelle du Mali, cent grammes de PDCO contient 5,33 g de protéines, $87 \mathrm{~g}$ de glucide, 0,67 g de lipides, 6,67 $\mathrm{mg}$ de fer, $1 \mathrm{mg}$ de zinc et $696,67 \mu \mathrm{g}$ équivalent rétinol (Barikmo et al., 2004).

Au Burkina Faso, à l'instar des autres pays d'Afrique de l'Ouest, les aliments complémentaires utilisés dans l'alimentation $\mathrm{du}$ jeune enfant en complément du lait maternel sont principalement des bouillies à base de céréales, pauvres en nutriments essentiels, donc contribuant peu à la couverture des besoins nutritionnels des jeunes enfants.

En revanche, les biscuits ont un potentiel considérable pour jouer un rôle de véhicule alimentaire dans la lutte contre la malnutrition. En effet, les biscuits sont des produits de grignotage de grande valeur énergétique élaborés à partir de farine de céréales, de matière grasse, de sucre et souvent additionné de poudre levante et divers autres ingrédients (O'Brien et al., 2003). Aussi, ce sont des aliments largement consommés par tous les âges, dans tous les pays, et particulièrement bien acceptés par les jeunes enfants, d'emploi facile et pouvant être consommés tels par les enfants ou rapidement délayés ou encore transformés en farine biscuitière pour la préparation de bouillie. La valeur nutritionnelle des biscuits peut être augmentée par complémentation ou fortification avec un grand nombre d'ingrédients nutritionnels, particulièrement riches en protéines, vitamines et minéraux (Aswathiet al., 2012).

La présente étude vise à étudier différents niveaux de substitution de la farine de sorgho par la poudre de feuilles séchées de moringa, la poudre de spiruline et la farine de patate douce à chair orange dans la formulation de biscuits de sorgho enrichis et l'impact sur la qualité nutritionnelle et sensorielle des biscuits en comparaison avec des biscuits témoins.

\section{MATERIEL ET METHODES}

Ressources végétales et ingrédients
sélectionnés et utilisés pour
l'enrichissement des biscuits de sorgho

Le principe de sélection des ressources végétales pour l'enrichissement des biscuits de sorgho repose sur leur disponibilité, leur potentiel nutritionnel, notamment riches en protéines, en fer, en zinc et ou en vitamine A, et leur acceptabilité. Les ressources végétales sélectionnées et utilisées dans les formulations de biscuits sont : la farine de sorgho (Sorghum bicolor) obtenue après décorticage et mouture des grains de sorgho effectués auprès du Centre de Transformation des Produits Agricoles (CTRAPA) à Ouagadougou; la poudre de feuilles séchées de Moringa (Moringa oleifera) achetée auprès de l'association des producteurs de la Sissili pour l'éco-gestion des ressources naturelles (APSERN); la poudre de spiruline (Spirulina platensis) achetée auprès de la ferme de production de Loumbila, ville située à $20 \mathrm{~km}$ de Ouagadougou;la farine de patate douce à chair orange (Ipomoea batatas) ou PDCO obtenue après mouture des cossettes de patate douce au niveau de l'atelier de production du Département Technologie Alimentaire (DTA) de l'Institut de Recherche en Sciences Appliquées et Technologies (IRSAT). Un complexe minéraux-vitaminique (CMV) obtenu auprès du programme Nutrifaso de Ouagadougou.

Les autres ingrédients comme la margarine, le sucre, les œufs, le sucre vanillé, le sel, la levure chimique ont été achetés dans une alimentation de la ville à Ouagadougou. La poudre de gomme arabique provient de l'atelier de production du DTA.

\section{Formulation et conditions de fabrication des biscuits de sorgho enrichis}

Une recette de base de biscuit de sorgho de type cookies développée par le 
DTA a été utilisée. Cette recette est composée des matières premières et ingrédients suivants : farine de sorgho $(52,9 \%)$, matière grasse végétale $(20 \%)$, sucre $(11 \%)$, œuf $(7,3 \%)$, poudre de gomme arabique $(4 \%)$, lait en poudre $(1,7 \%)$, poudre levante $(1,7 \%)$, sucre vanillé $(1,3 \%)$, sel $(0,1 \%)$. Cette composition constitue le témoin non enrichi. Pour les biscuits enrichis, une partie de la farine de sorgho était substituée par la poudre de feuilles séchées de moringa, la poudre de spiruline, la farine de patate douce à chair orange ou les CMV et les autres ingrédients étaient maintenues fixes. Pour chaque complément nutritionnel, trois niveaux de substitution de la farine de sorgho ont été retenus. Ils étaient de $4 \%, 6 \%$ et $8 \%$ pour la spiruline; $10 \%, 15 \%$ et $20 \%$ pour le moringa; $18 \%, 21 \%$ et $30 \%$ pour la PDCO; $0,26 \%$, $0,29 \%$ et $0,31 \%$ pour le CMV $(0,26 \%$ est le niveau de fortification des farines infantiles burkinabè fixé par Nutrifaso pour se rapprocher des recommandations nutritionnelles). Ces taux de substitution ont été choisis en tenant compte des normes de spécifications techniques sur les biscuits, des objectifs nutritionnels visés et des caractéristiques organoleptiques des produits finaux.

\section{Technologie de fabrication des biscuits de sorgho enrichis}

La technologie de fabrication des biscuits de sorgho enrichis développée au laboratoire par le DTA (Figure 1) comprend les principales étapes suivantes :

Pesée des matières premières et ingrédients, mélange-pétrissage progressif des ingrédients selon l'ordre de la recette, mise en forme des pâtons, cuisson des pâtons, refroidissement, conditionnement.

\section{Analyses physico-chimiques nutritionnelles \\ Teneurs en eau}

Les teneurs en eau des échantillons ont été déterminés par pesée différentielle avant et après passage à l'étuve à $130{ }^{\circ} \mathrm{C}$ pendant 2 heures selon la norme française (NF V 03707, 2000).

\section{Teneurs en lipides}

Les teneurs en lipides des échantillons ont été déterminées par la méthode d'extraction au Soxhlet selon la norme internationale (ISO 659 : 1998), avec l'hexane comme solvant.

\section{Teneurs en protéines totales}

Les teneurs en protéines totales ont été déterminées par la méthode de Kjeldahl selon la norme française (NF V03-050 : 1970).

\section{Teneurs en cendres totales}

Les teneurs en cendres ont été déterminées par incinération dans un four à moufle (Nabertherm®) à $550{ }^{\circ} \mathrm{C}$ pendant 4 heures selon la norme internationale (ISO 2171 : 2007).

\section{Teneurs en glucides totaux}

Les teneurs en glucides totaux ont été déterminées par différence (Egan et al., 1981) selon la formule: Teneur en glucides totaux $(\%)=100-[$ teneur en eau $(\%)+$ teneur en protéine $(\%)+$ teneur en lipide $(\%)+$ teneur en cendre $(\%)]$.

\section{Valeur énergétique}

La valeur énergétique a été calculée en utilisant les coefficients d'Atwater et Benedict (1899) selon la formule suivante: Valeur énergétique $(\mathrm{Kcal} / 100 \mathrm{~g})=$ teneur en glucides $(\%) \times 4(\mathrm{Kcal})+$ teneur en protéines $(\%) \times 4$ $(\mathrm{Kcal})+$ teneur en lipides $(\%) \times 9(\mathrm{Kcal})]$

\section{Teneurs en fer et en zinc}

Les teneurs en fer et zinc ont été déterminées par spectrométrie d'absorption atomique à flamme selon la méthode AOAC (2012).

\section{Evaluation sensorielle}

L'évaluation sensorielle des biscuits de sorgho enrichis a été réalisée avec un panel de 24 personnes entraînées (méthode modifiée de Awasthi et al., 2012; Tshite et al., 2015) utilisant le test d'acceptabilité avec l'épreuve de classement par rang sur une échelle hédonique de 5 points. Les biscuits de sorgho enrichis obtenus des différentes formulations 
au moringa, à la spiruline et à la PDCO ont été évaluées pour différents caractéristiques de qualité tels que : apparence, couleur, texture, consistance, odeur, parfum, arôme, goût. Une analyse de variance a été réalisée pour apprécier le degré de significativité des valeurs obtenues.

\section{Analyse statistique des données}

Toutes les analyses ont été effectuées en double. Les paramètres à l'étude ont été examinés à l'aide de statistiques descriptives (moyennes, écarts types, fréquences). Les données ont été soumises à une analyse de variance (ANOVA) à l'aide du logiciel SPSS $^{\text {TM }}$ Statistics version 20.0 (IBM,USA) et la comparaison des moyennes a été réalisée selon le test de DUNCAN au seuil de $5 \%$.

\section{RESULTATS}

\section{Composition globale des ingrédients nutritionnels}

Les ingrédients nutritionnels utilisés dans la fabrication des biscuits de sorgho enrichis ont été caractérisés au plan physicochimique. La spiruline a les teneurs en protéines les plus importantes, avec 52,5\%, soit deux fois plus élevées que celle de la poudre de feuilles de moringa $(24,7 \%)$, cinq fois la farine de sorgho $(10,4 \%)$ et dix fois environ la farine de PDCO $(4,9 \%)$. Les teneurs en lipides de la poudre de moringa et de la spiruline sont comparables, respectivement de $7,3 \%$ et $7,1 \%$ et plus importantes que celle de la farine de sorgho $(2,1 \%)$ et de la farine de patate douce à chair orange $(0,6 \%)$. De même, les teneurs en zinc $\mathrm{du}$ moringa $(4,2 \mathrm{mg} / 100 \mathrm{~g}$ de $\mathrm{MS})$ et de la spiruline (4,2 mg/100g de MS) sont deux fois supérieures à celles de la farine de sorgho $(1,9$ $\mathrm{mg} / 100 \mathrm{~g}$ de MS) et trois fois plus élevées que celle de la farine de PDCO $(1,4 \mathrm{mg} / 100 \mathrm{~g}$ de MS). Quant à la teneur en fer, les plus fortes concentrations sont observées avec la spiruline $(124,4 \mathrm{mg} / 100 \mathrm{~g}$ de $\mathrm{MS})$, suivie du moringa $(56,8 \mathrm{mg} / 100 \mathrm{gMS})$ qui en contient deux fois moins et pour le sorgho et la PDCO, respectivement de $14,4 \mathrm{mg} / 100 \mathrm{~g}$ de $\mathrm{MS}$ et $12,1 \mathrm{mg} / 100 \mathrm{~g}$ de $\mathrm{MS}$.
Déficits nutritionnels de la recette de base

En considérant les apports théoriques en nutriments essentiels (vitamine A, fer, zinc, protéines) des différents constituants de la recette de base établie pour la fabrication des biscuits de sorgho, et tenant compte des recommandations nutritionnelles du CODEX ALIMENTARIUS (1991) pour les enfants d'âge préscolaire, notamment de 6 à 23 mois, une détermination des manques à couvrir a été calculée (Tableau 1). Ce Tableau 1 montre que les teneurs complémentaires en nutriments à apporter par les ingrédients nutritionnels (moringa, spiruline, patate douce à chaire orange) à partir de $100 \mathrm{~g}$ de biscuit en vue de rencontrer les besoins journaliers sont de $8,3 \mathrm{mg}$ de fer, 8,6 $\mathrm{mg}$ de zinc, $365 \mu \mathrm{g}$ équivalent rétinol de vitamine $\mathrm{A}$ et $7,8 \mathrm{~g}$ de protéines.

Le Tableau 2 donne les taux d'incorporation des ingrédients nutritionnels calculés à partir des besoins journaliers en fer, zinc et vitamine A. Ce tableau montre que pour assurer les besoins journaliers en fer, en zinc en vitamine $\mathrm{A}$ et en protéines à partir de $100 \mathrm{~g}$ de biscuit de sorgho enrichis, il faudra incorporer théoriquement dans les formulations respectivement $11 \mathrm{~g}, 232 \mathrm{~g}, 4 \mathrm{~g}$ et $19 \mathrm{~g}$ de spiruline ; $30 \mathrm{~g}, 297 \mathrm{~g}, 7 \mathrm{~g}$ et $34 \mathrm{~g}$ de poudre de moringa, puis $188 \mathrm{~g}, 101 \mathrm{~g}, 57 \mathrm{~g}$ et $153 \mathrm{~g}$ de farine de patate douce.

\section{Qualité nutritionnelle des différentes formulations de biscuit de sorgho enrichis}

Au total, 13 formulations de biscuit de sorgho incluant les biscuits témoin (sans enrichissement et enrichis aux CMV) ont été réalisées. Le Tableau 3 donne les teneurs des principaux macronutriments déterminés pour les pâtons (pâtes non cuites) et les biscuits. Dans les pâtons, les taux d'humidité sont significativement $(\mathrm{p}<0,05)$ plus élevés avec les pâtons au moringa $(21,3 \pm 0,7 \%$ à $21,7 \pm 0,3 \%)$ et à la spiruline $(20,3 \pm 3,2 \%$ à $20,8 \pm 2,3 \%$ ) comparativement aux pâtons à la PDCO $(18,1 \pm 0,8 \%$ à $20,5 \pm 1,8 \%)$ et au CMV $(18,8 \pm 0,7 \%$ à $19,2 \pm 0,5 \%)$. Dans les biscuits, les taux d'humidité sont plus faibles compris 
entre $1,8 \pm 0,6 \%$ (CMV) et $7,9 \pm 1,1 \%$ (moringa).

Les teneurs en protéines des pâtons et des biscuits sont quasiment équivalentes pour une même formulation. Elles varient entre $5,7 \pm 0,0 \%$ et $12,0 \pm 0,3 \%$ pour les pâtons et entre $5,6 \pm 1,4 \%$ et $11,7 \pm 0,7 \%$ pour les biscuits. L'analyse de variance montre que les teneurs en protéines des pâtons et des biscuits de sorgho enrichis à la spiruline et au moringa sont significativement $(\mathrm{p}<0,05)$ plus élevées que celles des biscuits enrichis à la PDCO, aux CMV et au biscuit témoin non enrichi (Tableau 3).

Pour les lipides, il n'y a pas de différence significative $(p>0,05)$ entre les teneurs des pâtons et celles des biscuits. Elles sont comprises entre $19,4 \pm 0,6 \%$ et $24,3 \pm 6,1 \%$ pour les pâtons et entre $19,5 \pm 4,6 \%$ et $24,1 \pm 2,3 \%$ pour les biscuits avec les plus importantes valeurs pour les biscuits au moringa.

Concernant les cendres, les valeurs sont similaires pour les pâtons et les biscuits pour une même formulation et varient entre $2,0 \pm 0,1 \%$ et $4,1 \pm 0,0 \%$ dans les pâtons et entre $2 \pm 0,0 \%$ et $4,1 \pm 0,1 \%$ dans les biscuits. Elles sont significativement plus élevées $(p<0,05)$ dans les pâtons et les biscuits de sorgho au moringa.

Les teneurs en glucides totaux des biscuits sont significativement augmentées $(\mathrm{p}<0,05)$. Elles passent entre $31,5 \pm 4,8 \%$ et $52,4 \pm 2,2 \%$ dans les pâtons à des teneurs variant entre $53,2 \pm 2,2 \%$ et $68,5 \pm 0,5 \%$ dans les biscuits. Toutefois, il n'y a pas de différence significative entre les teneurs en glucides des biscuits.

Les valeurs énergétiques calculées pour toutes les formulations montrent que celles des pâtons sont significativement plus élevées $(\mathrm{p}<0,05)$, exceptés pour le biscuit témoin. En effet, les valeurs énergétiques sont comprises entre $440,9 \pm 28,6 \mathrm{Kcal} / 100 \mathrm{~g}$ et $518,9 \pm 7,6 \mathrm{Kcal} / 100 \mathrm{~g}$ pour les pâtons et entre $469,0 \pm 3,9 \mathrm{Kcal} / 100 \mathrm{~g}$ et $491,0 \pm 22 \mathrm{Kcal} / 100 \mathrm{~g}$ pour les biscuits. Aucune différence significative n'est observée entre les valeurs énergétiques des biscuits de sorgho enrichis avec ceux des biscuits témoin.

\section{Evolution des teneurs en zinc et en fer des biscuits à base de sorgho enrichis}

Les teneurs en fer et en zinc des pâtons et des biscuits de sorgho sont montrées dans la Figure 2. Concernant le fer, les teneurs sont significativement augmentées $(\mathrm{p}<0,05)$ avec l'enrichissement à l'opposé du zinc, exception pour celui au CMV. Les teneurs en fer et zinc varient respectivement entre $8,3 \pm 0,3 \mathrm{mg} / 100 \mathrm{~g}$ et $33,6 \pm 3,7 \mathrm{mg} / 100 \mathrm{~g}$ de $\mathrm{MS}$, puis entre $1,9 \pm 0,0$ et $19,0 \pm 0,5 \mathrm{mg} / 100 \mathrm{~g}$ de MS dans les pâtons tandis que dans les biscuits elles sont comprises entre $8,6 \pm 0,6 \mathrm{mg} / 100 \mathrm{~g}$ de $\mathrm{MS}$ et $22,9 \pm 2,7 \mathrm{mg} / 100 \mathrm{~g}$ de $\mathrm{MS}$, et entre $1,9 \pm 0,1$ $\mathrm{mg} / 100 \mathrm{~g}$ et $18,7 \pm 1,3 \mathrm{mg} / 100 \mathrm{~g}$ de $\mathrm{MS}$, avec les plus importantes valeurs pour les biscuits enrichis aux CMV. Cependant, seul l'enrichissement au CMV permet d'augmenter significativement les teneurs en fer et zinc des biscuits de sorgho. Les différences de valeurs entre les pâtons et les biscuits montrent des pertes au cours de la cuisson qui varient entre 12 et $47,4 \%$ pour le fer et entre 4,5 et $27,5 \%$ pour le zinc.

\section{Qualité sensorielle et acceptabilité des biscuits enrichis}

Les résultats du test de comparaison par paire pour les trois niveaux d'enrichissement de chaque ingrédient nutritionnel (spiruline, moringa, PDCO) sont présentés dans le Tableau 4. Il n'y a pas une différence d'acceptabilité significative $(\mathrm{p}>0,05)$ entre les biscuits de sorgho enrichis à $4 \%$ et $6 \%$ de spiruline et entre $6 \%$ et $8 \%$ de spiruline mais seulement entre 4 et $8 \%$ où on note une forte intensité de la coloration des biscuits avec l'augmentation des quantités de spiruline. Avec la PDCO, la différence d'acceptabilité entre les biscuits enrichis à $18 \%, 21 \%$ et $30 \%$ n'est pas significative. En revanche, avec le moringa, la différence d'acceptabilité entre les biscuits de sorgho 
enrichis à $10 \%, 15 \%$ et $20 \%$ de moringa est nette et très perceptible. Tout comme la spiruline, une augmentation du taux d'incorporation du moringa entraîne des changements de couleur et de goût des biscuits, qui ne sont pas bien appréciés des consommateurs. Les biscuits de sorgho enrichis ont obtenus une bonne appréciation des dégustateurs pour les plus faibles niveaux d'incorporation, soit $4 \%$ de spiruline, $10 \%$ de moringa et $18 \%$ de PDCO.

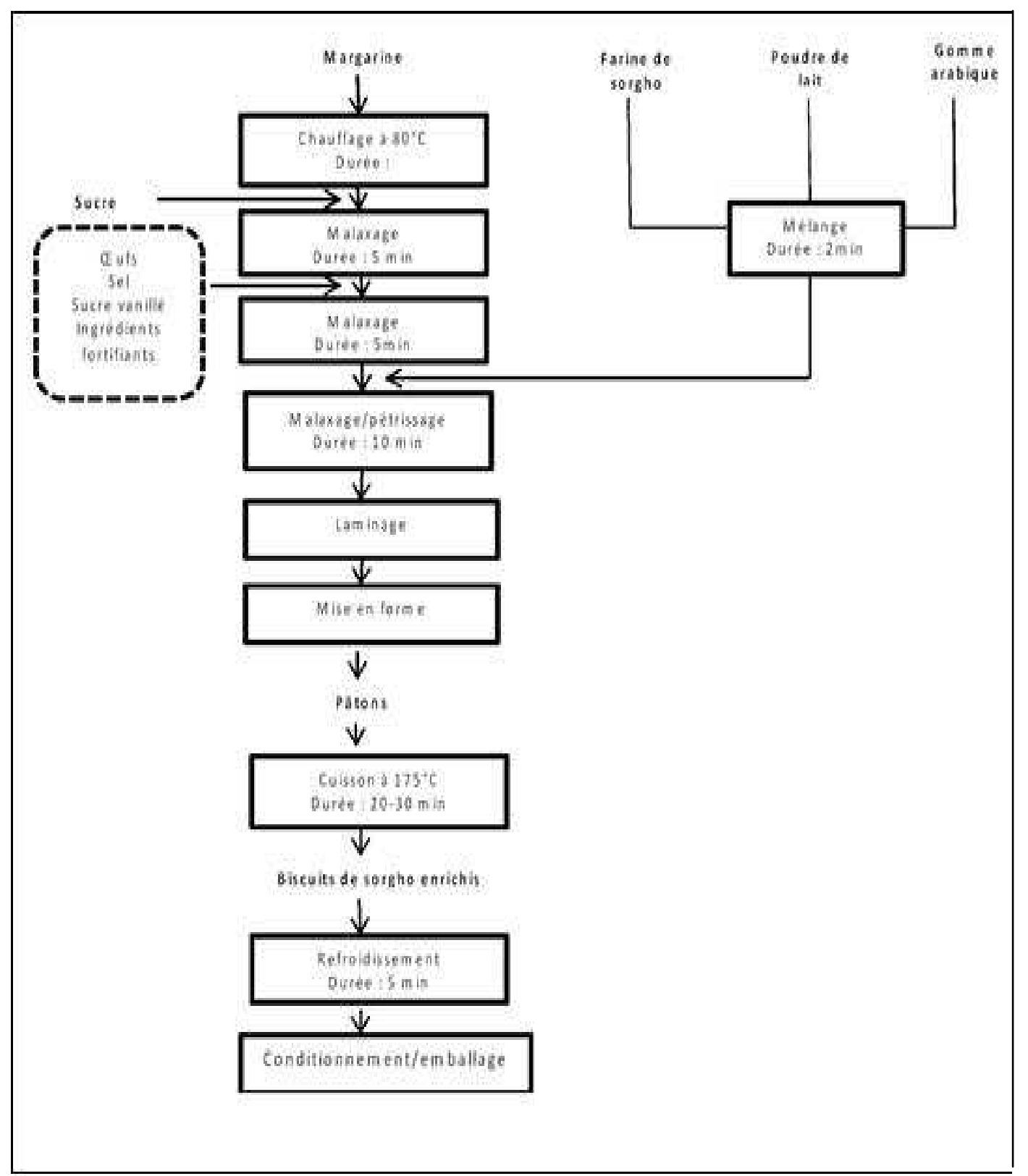

Figure 1: Etapes de fabrication des biscuits de sorgho enrichis et conditions opératoires. 


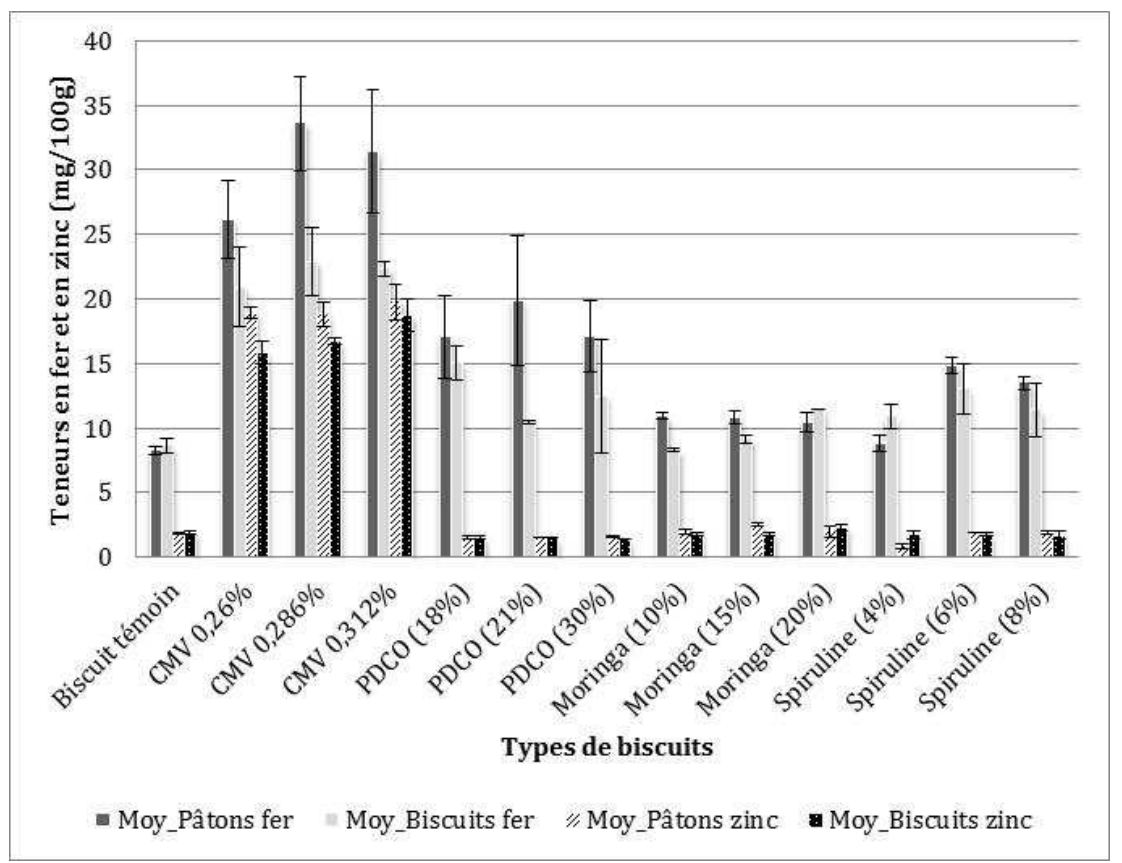

Figure 2: Teneurs en fer et zinc des pâtons de biscuits de sorgho enrichis.

Tableau 1 : Calcul des besoins en nutriments essentiels manquants.

\begin{tabular}{lccc}
\hline & $\begin{array}{c}\text { Apport journalier } \\
\text { recommandé (AJR) } \\
\text { pour les enfants de 6 à } \\
\text { 23 mois }\end{array}$ & $\begin{array}{c}\text { Apport nutritionnel de la recette } \\
\text { de base pour 100 g de biscuit } \\
\text { de sorgho }\end{array}$ & $\begin{array}{c}\text { Manque à } \\
\text { couvrir }\end{array}$ \\
\hline Fer $(\mathrm{mg})$ & 12 & & \\
Zinc (mg) & 10 & 3,7 & 8,3 \\
$\begin{array}{l}\text { Vitamine } \quad \mathrm{A} \quad(\mu \mathrm{g} \\
\text { équivalent rétinol) }\end{array}$ & 400 & 1,4 & 8,6 \\
Protéines (g) & 15 & 35,4 & 365 \\
\hline
\end{tabular}

Tableau 2: Calcul des taux d'incorporation des ingrédients fortifiants dans les formulations de biscuit de sorgho enrichis.

Quantité de chaque ingrédient fortifiant pour la couverture des besoins journaliers en fer, zinc et vitamine $A$

\begin{tabular}{lccc}
\hline $\begin{array}{l}\text { Quantité de nutriments à } \\
\text { apporter pour couvrir les besoins } \\
\text { nutritionnels journaliers }\end{array}$ & Spiruline $(\mathrm{g})$ & Moringa $(\mathrm{g})$ & $\begin{array}{c}\text { Patate douce à chair } \\
\text { orange }(\mathrm{g})\end{array}$ \\
\hline Fer $(8,3 \mathrm{mg})$ & 11 & 30 & 188 \\
Zinc $(8,6 \mathrm{mg})$ & 232 & 297 & 101 \\
Vitamine A $(365 \mu \mathrm{g}$ équivalent & 4 & 7 & 57 \\
rétinol) & 19 & 34 & 153 \\
Protéines $(7,8 \mathrm{~g})$ & & & \\
\hline
\end{tabular}


L. T. SONGRE-OUATTARA et al /Int. J. Biol. Chem. Sci. 10(4): 1651-1665, 2016

Tableau 3 : Composition globale des pâtons et des biscuits de sorgho enrichis (\%MS).

\begin{tabular}{|c|c|c|c|c|c|c|c|c|c|c|c|c|c|c|}
\hline & & & \multicolumn{2}{|c|}{ Humidité (g) } & \multicolumn{2}{|c|}{ Lipides (g) } & \multicolumn{2}{|c|}{ Protéines (g) } & \multicolumn{2}{|c|}{ Cendres (g) } & \multicolumn{2}{|c|}{ Glucides (g) } & \multicolumn{2}{|c|}{$\begin{array}{l}\text { Densité énergétique } \\
\text { (Kcal/100g) }\end{array}$} \\
\hline & & & Pâtons & Biscuits & Pâtons & Biscuits & Pâtons & Biscuits & Pâtons & Biscuits & Pâtons & Biscuits & Pâtons & Biscuits \\
\hline \multicolumn{3}{|c|}{ Recette de base } & $18,1 \pm 0,7$ & $3,4 \pm 1,1$ & $24,3 \pm 6,1$ & $20,9 \pm 1,4$ & $6,2 \pm 0,6$ & $6,9 \pm 0,5$ & $2,0 \pm 0,1$ & $2 \pm 0,0$ & $49,5 \pm 6,9$ & $66,8 \pm 2,3$ & $440,9 \pm 28,6$ & $483,2 \pm 7,7$ \\
\hline \multirow{9}{*}{ 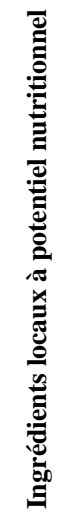 } & \multirow{3}{*}{ Spiruline } & $4 \%$ & $20,3 \pm 3,2$ & $4,1 \pm 2,4$ & $20,7 \pm 1,8$ & $19,9 \pm 2,4$ & $9,6 \pm 0,8$ & $9,2 \pm 0,9$ & $2,4 \pm 0,0$ & $2,4 \pm 0,0$ & $46,1 \pm 5,0$ & $64,3 \pm 3,6$ & $498,2 \pm 5,4$ & $473,4 \pm 8,2$ \\
\hline & & $6 \%$ & $20,5 \pm 1,7$ & $4,5 \pm 0,8$ & $23,4 \pm 4,7$ & $20,8 \pm 2,3$ & $11,4 \pm 0,1$ & $10,5 \pm 0,8$ & $2,7 \pm 0,1$ & $2,7 \pm 0,1$ & $39,4 \pm 0,1$ & $61,6 \pm 2,4$ & $518,9 \pm 7,6$ & $475,3 \pm 8,3$ \\
\hline & & $8 \%$ & $20,8 \pm 2,3$ & $5,3 \pm 1,4$ & $20,5 \pm 1,4$ & $20,7 \pm 1,2$ & $12,0 \pm 0,3$ & $11,7 \pm 0,7$ & $3,0 \pm 0,1$ & $3,0 \pm 0,1$ & $43,1 \pm 1,2$ & $59,4 \pm 1,7$ & $493,1 \pm 6,5$ & $470,2 \pm 6,5$ \\
\hline & \multirow{3}{*}{ Moringa } & $10 \%$ & $21,3 \pm 0,7$ & $5,6 \pm 3,4$ & $20,7 \pm 1,3$ & $22,5 \pm 2,4$ & $8,5 \pm 0,1$ & $8,9 \pm 0,3$ & $3,0 \pm 0,1$ & $3,0 \pm 0,0$ & $46,9 \pm 2,4$ & $60,0 \pm 3,9$ & $489,3 \pm 8,2$ & $477,9 \pm 17,4$ \\
\hline & & $15 \%$ & $21,4 \pm 0,2$ & $6,8 \pm 2,1$ & $23,3 \pm 2,2$ & $23,5 \pm 1,6$ & $9,4 \pm 0,5$ & $9,4 \pm 0,6$ & $3,6 \pm 0,0$ & $3,6 \pm 0,1$ & $42,3 \pm 3,7$ & $56,8 \pm 1,2$ & $502,1 \pm 15,2$ & $475,9 \pm 14,0$ \\
\hline & & $20 \%$ & $21,7 \pm 0,3$ & $7,9 \pm 1,1$ & $24,1 \pm 2,4$ & $24,1 \pm 2,3$ & $10,0 \pm 0,3$ & $10,7 \pm 1,0$ & $4,1 \pm 0,0$ & $4,1 \pm 0,1$ & $40,4 \pm 3,2$ & $53,2 \pm 2,2$ & $501,5 \pm 16,9$ & $482,9 \pm 20,4$ \\
\hline & \multirow{3}{*}{ PDCO } & $18 \%$ & $20,5 \pm 1,8$ & $4,3 \pm 2,1$ & $22,1 \pm 2,2$ & $20,2 \pm 3,5$ & $6,3 \pm 0,2$ & $6,2 \pm 1,2$ & $2,4 \pm 0,0$ & $2,3 \pm 0,1$ & $50,0 \pm 2,4$ & $67,0 \pm 2,8$ & $494,7 \pm 4,1$ & $474,6 \pm 25,1$ \\
\hline & & $21 \%$ & $18,1 \pm 0,8$ & $5,9 \pm 0,8$ & $19,4 \pm 0,6$ & $20,4 \pm 0,2$ & $6,4 \pm 0,7$ & $6,3 \pm 0,9$ & $2,4 \pm 0,0$ & $2,4 \pm 0,1$ & $54,0 \pm 0,2$ & $65,0 \pm 0,4$ & $485,5 \pm 1,4$ & $469,0 \pm 3,9$ \\
\hline & & $30 \%$ & $18,8 \pm 0,2$ & $5,3 \pm 1,3$ & $21,1 \pm 1,9$ & $21,1 \pm 2,4$ & $5,7 \pm 0,0$ & $5,6 \pm 1,4$ & $2,6 \pm 0,0$ & $2,7 \pm 0,3$ & $52,4 \pm 2,2$ & $65,3 \pm 2,5$ & $492,0 \pm 11,4$ & $473,5 \pm 9,0$ \\
\hline \multirow{3}{*}{ 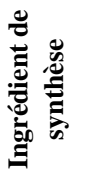 } & \multirow{3}{*}{ CMV } & $0,26 \%$ & $18,8 \pm 0,7$ & $1,8 \pm 0,6$ & $21,7 \pm 0,5$ & $20,8 \pm 1,1$ & $7,1 \pm 0,3$ & $6,8 \pm 0,3$ & $2,1 \pm 0,2$ & $2,0 \pm 0,2$ & $50,4 \pm 0,4$ & $68,5 \pm 0,5$ & $499,9 \pm 3,2$ & $488,7 \pm 7,9$ \\
\hline & & $0,28 \%$ & $19,0 \pm 0,7$ & $3,1 \pm 1,1$ & $21,5 \pm 3,9$ & $22,3 \pm 4,6$ & $6,3 \pm 2,3$ & $7,2 \pm 0,4$ & $2,2 \pm 0,2$ & $2,1 \pm 0,1$ & $33,6 \pm 3,7$ & $65,3 \pm 4,7$ & $498,4 \pm 20,3$ & $491,0 \pm 22,0$ \\
\hline & & $0,31 \%$ & $19,2 \pm 0,5$ & $3,7 \pm 0,6$ & $22,3 \pm 1,7$ & $19,5 \pm 4,6$ & $7,6 \pm 0,2$ & $7,3 \pm 0,2$ & $2,2 \pm 0,2$ & $2,2 \pm 0,1$ & $31,5 \pm 4,8$ & $67,4 \pm 5,0$ & $502,4 \pm 9,0$ & $474,0 \pm 22,1$ \\
\hline
\end{tabular}


L. T. SONGRE-OUATTARA et al/Int. J. Biol. Chem. Sci. 10(4): 1651-1665, 2016

Tableau 4: Comparaison par paire des trois niveaux d'enrichissement pour la spiruline, le moringa et la PDCO.

\begin{tabular}{|c|c|c|c|c|c|c|c|c|c|}
\hline \multirow{2}{*}{$\begin{array}{l}\text { Formulations } \\
\text { Taux d'incorporation }\end{array}$} & \multicolumn{3}{|c|}{ Spiruline } & \multicolumn{3}{|c|}{ Moringa } & \multicolumn{3}{|c|}{ PDCO } \\
\hline & $4 \%$ & $6 \%$ & $8 \%$ & $10 \%$ & $15 \%$ & $20 \%$ & $18 \%$ & $21 \%$ & $30 \%$ \\
\hline Total des scores & 40 & 46 & 60 & 29 & 49 & 66 & 45 & 53 & 46 \\
\hline $\begin{array}{l}\text { Moyennes des scores } \\
\text { attribués }\end{array}$ & $1,67^{\mathrm{a}}$ & $1,92^{\mathrm{ab}}$ & $2,5^{\mathrm{b}}$ & $1,21^{\mathrm{a}}$ & $2,04^{\mathrm{b}}$ & $2,75^{\mathrm{c}}$ & $1,88^{\mathrm{a}}$ & $2,21^{\mathrm{ab}}$ & $1,92^{\mathrm{a}}$ \\
\hline Rang & $1^{\mathrm{er}}$ & $2^{\text {ème }}$ & $3^{\text {ème }}$ & $1^{\mathrm{er}}$ & $2^{\text {ème }}$ & $3^{\text {ème }}$ & $1^{\mathrm{er}}$ & $3^{\text {ème }}$ & $2^{\text {ème }}$ \\
\hline
\end{tabular}




\section{DISCUSSION}

Les résultats de caractérisation nutritionnelle des treize formulations de biscuit de sorgho enrichis avec la spiruline, le moringa, la PDCO ou les CMV et non enrichis montrent des variations significatives pour les taux d'humidité en concordance avec ceux de Okpala et al. (2012) sur des biscuits faits de mélanges de farines à base de pois sucré, farine de sorgho germée et de coco et ceux de Tshite et al., (2015) sur des farines précuites à base de maïs et de soja. Ces derniers ont montré que les taux d'humidité dans les biscuits sont généralement faibles, moins de $10 \%$, ce qui conditionne une longue conservation. De même, les variations importantes observées pour les teneurs en protéines de ces différentes formulations sont en accord avec ceux de Okpala et al. (2012) qui indiquent qu'elles varient entre $4,85 \%$ et $19,89 \%$ pour les biscuits de mélanges de farines.

L'enrichissement avec la spiruline et le moringa entraîne une augmentation des teneurs en protéines, respectivement entre 33 et $70 \%$ et entre 29 et $55 \%$, consécutif aux teneurs élevées de ces additifs en protéines. Cependant, pour les niveaux d'enrichissement étudiés, les teneurs en protéines de ces biscuits de sorgho à la spiruline et au moringa sont inférieures à $15 \%$, qui représentent l'apport journalier recommandé pour les enfants d'âge compris entre 6 et 23 mois par le CODEX ALIMENTARIUS. Aussi, comparés aux farines infantiles généralement enrichies avec des légumineuses (haricot, soja, arachide) localement fabriquées au Burkina Faso (Misola, Natavie, Céréalor, Nutrilac, Vitazom Yonhanma) qui ont entre 15 et 16,8\% de protéines, ces biscuits de sorgho enrichis sont de teneurs en protéines améliorées mais cependant en deçà de ces derniers. Les teneurs en cendres et en lipides des différentes formulations sont plus élevées que celles des farines infantiles locales (9 à 10\% de lipides) et celles rapportées par Rai et al. (2014) qui ont montré que des biscuits de plusieurs combinaison de céréales sans gluten (sorgho, riz, mil) avaient un maximum de teneur en lipides de $19,2 \%$ et de cendre de 1,6\%. Ainsi l'enrichissement avec le moringa, la spiruline et la PDCO n'a pas permis une augmentation significative des teneurs en lipides des biscuits $\mathrm{du}$ fait qu'ils sont de faibles teneurs inférieures à $8 \%$. Cependant, pour l'ensemble des formulations, les teneurs en lipides obtenues répondent aux apports journaliers préconisés, soit 10 à $25 \mathrm{~g}$ par le CODEX ALIMENTARIUS chez l'enfant de 6 à 24 mois. Concernant les valeurs énergétiques, elles sont comparables pour les différentes formulations avec un équilibre nutritionnel différent, particulièrement riches en protéines et donc en acides aminés essentiels pour les biscuits au moringa et à la spiruline. Ces valeurs sont proches de celles rapportées par Rai et al. (2014) et supérieures à la valeur minimale de $400 \mathrm{kcal} / 100 \mathrm{~g}$ telle que recommandée pour le jeune enfant selon le CODEX ALIMENTARIUS 1991. S'agissant des minéraux, l'enrichissement au moringa, à la spiruline, à la PDCO et au CMV ont contribué à augmenter les teneurs en fer des biscuits de sorgho mais seuls les CMV ont permis d'améliorer de manière efficiente et significative le contenu en fer et en zinc des biscuits de sorgho au-delà des apports journaliers recommandés du CODEX ALIMENTARIUS qui sont respectivement de $12 \mathrm{mg}$ et $10 \mathrm{mg}$. Par ailleurs, l'augmentation des teneurs en fer est plus importante avec la PDCO qu'avec le moringa et la spiruline et cela pourrait être due à des contaminations avec le matériel et les équipements durant la transformation de la patate douce en cossette et en farine. 
Effet de la cuisson sur la qualité nutritionnelle et sensorielle des biscuits enrichis

La cuisson des pâtons a entraîné une perte importante des teneurs en eau permettant ainsi de mieux les conserver. Aussi, cette réduction en eau se traduit par l'accroissement des teneurs en glucides dans les biscuits. En revanche, la cuisson n'a pas eu d'effet notable sur les teneurs en protéines, en lipides, et en cendres des biscuits. Pour les minéraux étudiés, la perte était plus importante avec le fer qu'avec le zinc durant la cuisson, ce qui permet de conclure que le fer est plus sensible à la chaleur. Ces résultats sont différents de ceux de Vautier et al. (2010) qui ont montré que la cuisson au four augmente significativement les teneurs en protéines, lipides, cendres, fer et zinc du rôti filet de porc $(+49 \%,+47 \%,+25 \%,+55 \%$, et $+42 \%$ respectivement). Ces différences pourraient être attribuées à la nature des substrats et à leurs caractéristiques physico-chimiques. Sur le plan organoleptique, les modifications ont porté sur la couleur, le goût, la texture et la couleur des biscuits.

\section{Acceptabilité des biscuits de sorgho enrichis}

Ces différentes formulations de biscuits de sorgho enrichis montrent des caractéristiques nutritionnelles similaires aux biscuits commercialisés ou développées à partir de mélange de farines. Pour l'acceptabilité des biscuits de sorgho enrichis à la PDCO, les difficultés de différenciation observées avec les trois niveaux d'enrichissement pourraient être expliquées par un mélange de saveur sucrée et d'amertume attribué à la farine de PDCO, tel que souligné par les dégustateurs. A cet effet, il a été observé que plus la quantité de PDCO est élevée plus un arrière-goût s'en dégage et plus le produit semble plus sucré. Avec le moringa, l'acceptabilité pour le faible niveau d'enrichissement est expliquée par les changements organoleptiques des biscuits, particulièrement la couleur plus foncée et le goût plus amer qui s'intensifient avec l'augmentation des taux d'incorporation du moringa. De manière similaire au moringa, l'augmentation des taux d'incorporation de la spiruline entraîne une intensification de la couleur verte des biscuits qui n'est pas bien accepté des dégustateurs. Ces résultats permettent de conclure que les biscuits de sorgho enrichis au moringa et à la spiruline sont acceptés des dégustateurs seulement à des niveaux d'enrichissement très faibles, soit de $4 \%$ pour la spiruline et $10 \%$ pour le moringa.

\section{Conclusion}

Cette étude a démontré la faisabilité de substituer la farine de sorgho par des ingrédients nutritionnels comme le moringa, la spiruline et la PDCO pour la production de biscuits de sorgho enrichis avec des teneurs en protéines, et en fer plus importantes et de qualité sensorielle acceptable. L'utilisation de ces ingrédients nutritionnels est une alternative peu coûteuse et accessible par rapport à celle de l'enrichissement par les CMV plus difficile à réaliser au Burkina Faso à cause de sa faible accessibilité par les acteurs de transformations. Ces résultats ouvrent une voie de valorisation des ressources végétales et les travaux pourront être approfondis à travers une étude de détermination de la durée de vie et du profil en acides aminés de ces produits.

\section{CONFLIT D'INTERET}

Les auteurs déclarent l'absence de conflit d'intérêt.

\section{CONTRIBUTIONS DES AUTEURS}

OSLT a conçu le sujet, dirigé les travaux, effectué les analyses statistiques et rédigé le manuscrit; GK a effectué les 
analyses biochimiques et a contribué à la rédaction du manuscrit; $\mathrm{SA}$ a codirigé les travaux et corrigé le manuscrit; $\mathrm{BF}$ a participé à la rédaction du manuscrit; $\mathrm{DB}$ a réalisé les orientations techniques et corrigé le manuscrit.

\section{REMERCIEMENTS}

Les auteurs remercient le programme Nutrifaso au Burkina Faso pour la fourniture des complexes minéraux vitaminiques.

\section{REFERENCES}

Anwar F, Latif S, Ashraf M, Gilani AH. 2007. Moringa oleifera: A Food Plant with Multiple Medicinal Uses: a review. Phytother Res, 21(1): 17-25. DOI: 10.1002/ptr.2023

AFNOR (Association Française de Normalisation). $1970 . \quad$ Directives générales pour le dosage de l'azote avec minéralisation selon la méthode de Kjedahl. Produits Agricoles Alimentaires, NF V 03-050.

AFNOR (Association Française de Normalisation). 2000. Détermination de la teneur en eau, méthode pratique. Céréales, Légumineuses, Produits Dérivés, NF V 03-707.

AOAC (Association of Official Agricultural Chemists). 2012. Official methods of analysis chemists, 19 the dn; (AOAC Arlington), Virginia, USA.

Atwater WO, Benedict FG. 1899. Experiments on the Metabolism of Matter and Energy in the Human Body. US Department of Agriculture: Washington D.C. Bulletin 69; 112p.

Awasthi I, Siraj P, Tripathi M, Tripathi V. 2012. Development of soy fortified high protein and high calorie supplementary biscuits. Indian J. Sci. Res., 3(1): 51-58.

Babadzhanov AS, Abdusamatova N, Yusupova F M, Faizullaeva N, Mezhlumyan LG, Malikova MK. 2004.
Chemical composition of Spirulina platensis cultivated in Uzbekistan. Chem. Nat. Compd., 40: 276-279.

Barikmo I, Ouattara F, Oshaug A. 2004. Food Composition Table for Mali. TACAM, research series N 9. Akershus University College, Oslo.

Belay A. 2002. The potential application of Spirulina (Arthrospira) as a nutritional and therapeutic supplement in health management. J. Am. Nutraceutical Assoc, 5: $27-48$.

Broin M. 2005. Nutritional composition of Moringa oleifera leaves. Moringa news, $5 \mathrm{pp}$.

Dillard CJ, German JB. 2000. Phytochemicals: nutraceuticals and human health: a review. J. Sci. Food Agric, 80: 1744-1756.

Diraman H, Koru E, Dibeklioglu H. 2009. Fatty acid profile of Spirulina platensis used as a food supplement. Isr. J. Aquacult-Bamid., 61: 134-142.

Egan H, Kirk RS, Sawyer R. 1981. Pearson's Chemical Analyses of Food ( $8^{\text {th }}$ edn). Churchill Livingstone: London-UK; 591p.

Habib MAB, Parvin M, Huntington TC, Hasan MR. 2008. A Review on Culture, Production and Use of Spirulina as Food for Humans and Feeds for Domestic Animals and Fish, FAO Fisheries and Aquaculture Circular. Food and Agriculture Organisation of the United Nations (FAO): Rome; 1-26.

Haskell MJ, Jamil KM, Hassan F, Peerson JM, Hossain ML, Fuchs GJ, Brown KH. 2004. Daily consumption of Indian spinach (Basella alba) or sweet potatoes has a positive effect on total-body vitamin A stores in Bangladeshi men. Am. J. Clin. Nutr., 80(3): 705-714.

Holman BWB, Malau-Aduli AEO. 2013. Spirulina as a livestock supplement and animal feed. J. Anim. Physiol. Anim. 
Nutr., 97: 615-623. DOI: 10.1111/j.14390396.2012.01328.x

INSD (Institut National de la Statistique et de la Démographie). 2012. Enquête Démographique et de Santé et à Indicateurs Multiples du Burkina Faso 2010. INSD : Calverton, Maryland, USA ; 501p.

ISO (International Standardization Organization). 1998. Détermination de la teneur en matière grasse selon la méthode d'extraction par Soxhlet. ISO 659.

ISO (International Standardization Organization). 2007. Dosage du taux de cendre par incinération à $550 \quad{ }^{\circ} \mathrm{C}$. Céréales, légumineuses et produits dérivés. ISO 2171.

Mawouma S, Mbofung CM. 2014. Usages alimentaires de Moringa oleifera dans la région de l'Extrême-Nord Cameroun. Int. J. Biol. Chem. Sci., 8(4): 1847-1852. DOI: http://dx.doi.org/10.4314/ijbcs.v8i4.42

Mensah P, Tomkins A. 2003. Household-level technologies to improve the availability and preparation of adequate and safe complementary foods. The United Nations University. Food Nutr. Bull., 24(1): 104-125.

Muhling M, Belay A, Whitton BA. 2005. Variation in fatty acid composition of Arthrospira (Spirulina) strains. J. Appl. Phycol., 17: 137-146. DOI: 10.1007/s10811-005-7213-9.

Ndong M, Wade S, Dossou N, Guiro AT, Gning RD. 2007. Valeur nutritionnelle du Moringa oleifera, étude de la biodisponibilité du fer, effet de l'enrichissement de divers plats traditionnels sénégalais avec la poudre des feuilles. Afric. J. Food. Agr. Nutri. Develop., 7(3) online 17p.

O'Brien CN, Champmemb O, Nexille DP, Kengh MK, Arendt EK. 2003. Effect of varying micro en capsulation process on the functionality hydrogenated vegetable fat in short dough biscuit. Food Res. Int., 36: 215-221.

Okpala L, Okoli1 E, Udensi E. 2013.Physicochemical and sensory properties of cookies made from blends of germinated pigeon pea, fermented sorghum, and cocoyam flours. Int. J. Food Sci. Nutr., 1(1): 8-14.doi: 10.1002/fsn3.2.

RaiS, Kaur A, Singh B. 2014. Quality characteristics of gluten free cookies prepared from different flour combinations. J. Food Sci. Technol., 51(4): 785-789. DOI 10.1007/s13197011-0547-1

Sanchez M, Bernal-Castillo J, Rozo C, Rodriguez I. 2003. Spirulina (Arthrospira): an edible microorganisma review. Univ. Sci., 8(1): 7-24.

Siddhuraju P, Becker K. 2003. Antioxidant properties of various solvent extracts of total phenolic constituents from three different agro-climatic origins of drumstick tree (Moringa oleifera Lam). J. Agric. Food Chem., 15: 2144-2155.

Tété-Bénissan A, Lawson-Evi KA, Kokou K, M Gbéassor. 2012. Effet de la poudre de feuilles de moringa oleiferalam sur l'évolution du profil de l'hémogramme des enfants malnutris au togo: évaluation chez les sujets hiv positifs. Afr. J. Food Agric. Nutr. Dev., 12(2): 6007-6026.

Thurber MD, Fahey JW. 2009. Adoption of Moringa oleifera to combat undernutrition viewed through the lens of the "Diffusion of Innovations" theory. Ecol. Food. Nutr., 48(3): 212-225. Doi: 10.1080/03670240902794598.

Tshite FN, Mulamba VT, Ndianabo MJT. 2015. Mise au point d'une farine précuite à base de maïs (Zea mays) et de soja (Glycine max) par la méthode traditionnelle. Int. J. Biol. Chem. Sci., 9(6): 2608-2622. DOI: http://dx.doi.org/10.4314/ijbcs.v9i6.8 
UNICEF (Fonds des Nations Unies pour l'enfance). 2009. Carences en Vitamines et Minéraux, Rapport sur les Progrès dans le monde. UNICEF ; 39p.

Van Jaarsveld PJV, Faber M, Tanumihardjo SA, Nestel P, Lombard CJ, Benadé AJ. 2005. Carotene-rich orange-fleshed sweet potato improves the vitamin A status of primary school children assessed with the modified-relative-dose-response test. Am. J. Clin. Nutr., 81(5): 1080-1087.

Vautier A, Carlier M, Martin JL, Gault E, Vendeuvre JL. 2010. Impact de la cuisson et de la température à cœur sur les valeurs nutritives du rôti filet de porc. Communication présentée aux $42^{\text {èmes }}$ Journées de la Recherche Porcine (2010). Techni Porc, 33(1): 15-16. 